\title{
Chronisch-persistierende Neurolues mit epileptischen Anfällen, distaler Neuropathie und kognitiver Einschränkung trotz wiederholter antibiotischer Therapie
}

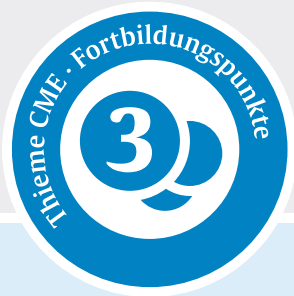

Matthias Karrasch ${ }^{1,2}$, Wolfgang Pfister ${ }^{1}$, Michael Baier ${ }^{1}$, Hubertus Axer ${ }^{3}$, Otto W. Witte ${ }^{3}$, Matthias Nürnberger ${ }^{3}$

1 Institut für Medizinische Mikrobiologie, Universitätsklinikum Jena

2 Institut für Klinische Chemie und Laboratoriumsdiagnostik, Universitätsklinikum Jena

3 Klinik für Neurologie, Universitätsklinikum Jena

\section{ZUSAMMENFASSUNG}

Eine 55-jährige Patientin stellte sich zur Abklärung eines generalisierten epileptischen Anfalls in der zentralen Notaufnahme unseres Universitätsklinikums vor. Im Rahmen der weiteren Untersuchungen fielen kognitive Einschränkungen und eine axonal-demyelisierende Polyneuropathie auf. Laborchemisch war initial lediglich eine leichte Pleozytose in der Liquoruntersuchung auffällig. Im Rahmen der weiteren Abklärung der klinischen Beschwerden und der Liquorpleozytose konnte bei der Patientin infektionsserologisch eine chronisch-persistierende Treponema-pallidum-Infektion gesichert werden. Die gleiche Diagnose konnte anschließend auch bei dem Lebenspartner der Patientin gestellt werden. Bei generalisierten epileptischen Anfällen, zunehmenden kognitiven Einschränkungen und axonal-demyelinisierender Polyneuropathie unklarer Genese sollte eine Neurolues differenzialdiagnostisch erwogen und eine Liquoruntersuchung durchgeführt werden.

\section{Einleitung}

Die Inzidenz der Syphilis als sexuell übertragbare Infektion steigt weltweit weiter an. Im Jahr 2017 wurden dem Robert-Koch-Institut 7476 Syphilis-Fälle aus Deutschland gemeldet, die Anzahl der Meldungen stieg im Vergleich zu 2016 um 4,2 \% [1]. Während der direkte Erregernachweis im Primäraffekt des Stadiums I der Syphilis mittels Dunkelfeldmikroskopie relativ einfach geführt werden kann, stellen Spätmanifestationen im Stadium II/III häufig eine diagnostische Herausforderung dar. Da humanpathogene Treponemen mit den üblichen Nährmedien schlecht kultivierbar sind, besteht die Methode der Wahl zur Laboratoriumsdiagnose der Syphilis aus dem Antikörpernachweis (TPPA-Suchtest, FTA-Abs Bestätigungstest, VDRL - Beurteilung der Aktivität der Infektion, spezifischer Antikörperindex). Die Therapie der Syphilis wurde erstmalig von Paul Ehrlich in die Klinik eingeführt. Bei Neurosyphilis muss zur Erreichung treponemozider Antibiotikaspiegel im Liquor über einen längeren Zeitraum mit Penicillinen und Cephalosporinen therapiert werden [2].

\section{Klinischer Fallbericht}

Eine 55-jährige Patientin stellte sich mit einem generalisierten epileptischen Anfall in der zentralen Notaufnahme unseres Universitätsklinikums zur weiteren Abklärung vor. Eigenanamnestisch bestand eine retrograde Amnesie für das Ereignis und es wurde zudem ein generalisierter Anfall vor 4 Jahren beschrieben. Eine regelmäßige Medikamenteneinnahme wurde verneint. Relevante Vorerkrankungen lagen nicht vor. Klinisch neurologisch fand sich bei Aufnahme eine wache, vollständig orientierte Patientin ohne fokalneurologisches Defizit. Laborparametrisch ergaben sich keine Auffälligkeiten. Die Entzündungswerte im Serum waren unauffällig; es fiel lediglich ein nitritnegativer Harnwegsinfekt mit Leukozyturie auf. Nach Abnahme einer Urinkultur wurde über 5 Tage kalkuliert mit Ciprofloxacin behandelt, worunter sich keine Besserung des Urinstatus zeigte. MR-tomografisch konnten bis auf eine Seitendifferenz der temporomesialen Strukturen in der koronaren Dünnschichtung keine Pathologien festgestellt werden. Das EEG zeigte ein unauffälliges Alpha-EEG ohne Hinweise für epilepsietypische Potenziale oder Zeichen neuronal gesteigerter Erregbarkeit. Langzeitblutdruckmessung, EKG und Schellong-Test ergaben keinen Hinweis auf Anomalien. Zur Komplettierung der Diagnostik wurde eine diagnostische Lumbalpunktion (LP1, > Tab. 1) durchgeführt.

Hierbei zeigte sich ein leicht erhöhtes Gesamtprotein $(658 \mathrm{mg} / \mathrm{l})$ und eine lymphozytäre Pleozytose (erhöhte Gesamtzellzahl 25 Zellen/ $\mu$ l, 89\% Lymphozyten und vereinzelt aktivierten Monozyten in der Zelldifferenzierung; Glukose 7,9 mmol/l, Laktat 1,4.), darunter aktivierte Formen mit größeren Kernen mit aufgelockerter Chromatinstruktur und perinukleär aufgehellten Zellsäumen ( $>$ Abb. 1). Weiterhin war eine leichte Blut-Liquor-Schrankenfunktionsstörung und eine intrathekale IgG- und IgM-Synthese sowohl rechnerisch als auch über den Nachweis oligoklonaler Banden im Liquor nachweisbar (oligoklonale Banden negativ im Serum). Aufgrund dieser Befundkonstellation war zu diesem Zeitpunkt ein chronisch-entzündlicher ZNS-Prozess wahrscheinlich. Es wurde daraufhin eine mikrobiologische Diagnostik im Serum und im Liquor initiiert. 
- Tab. 1 Ergebnisse der Liquorpunktionen (LP) beider Patienten im Verlauf. Über einen Zeitraum von 1,5 Jahren wurden insgesamt 5 LP bei der Patientin und 3 LP beim Patienten durchgeführt. Die Liquorpunktion fand für beide Patienten am jeweils gleichen Tag statt. (Referenzbereiche lumbal: Gesamtprotein: 150-400 mg/l; Gesamtzellzahl im Liquor: 0-5 Zellen/ $\mu$ l, Treponema pallidum VDRL Titer <2, TPPA-AK-Index Liquor<2); VDRL: Venereal Disease Research Laboratory (Cardiolipin-Antikörper, Marker der Entzündungsaktvität); TPPA-Al: Treponema-pallidum-Antikörper-Index

\begin{tabular}{|c|c|c|c|c|}
\hline Liquorpunktion (LP) & Gesamtprotein [mg/l] & $\begin{array}{l}\text { Zellzahl [Zellen/ul] } \\
\text { (\% Diff) }\end{array}$ & VDRL & TPPA AI \\
\hline \multicolumn{5}{|l|}{ Ehefrau } \\
\hline LP 1 & 658 & $\begin{array}{l}25 \\
\text { (89\% Lymphozyten) }\end{array}$ & 8 & 5,3 \\
\hline LP 2 & 614 & $\begin{array}{l}9 \\
\text { (92\% Lymphozyten) }\end{array}$ & 8 & 2,5 \\
\hline LP 3 & 477 & $\begin{array}{l}5 \\
\text { (92\% Lymphozyten) }\end{array}$ & 4 & 10,3 \\
\hline LP 4 & 561 & $\begin{array}{l}1 \\
\text { (keine Differenzierung) }\end{array}$ & 2 & 17,4 \\
\hline LP 5 & 514 & $\begin{array}{l}1 \\
\text { (keine Differenzierung) }\end{array}$ & 2 & 9,7 \\
\hline \multicolumn{5}{|l|}{ Ehemann } \\
\hline LP 1 & 336 & $\begin{array}{l}2 \\
\text { (94\% Lymphozyten) }\end{array}$ & 2 & 19,6 \\
\hline LP 2 & 718 & $\begin{array}{l}25 \\
\text { (34\% Lymphozyten, 56\% } \\
\text { Granulozyten, massenhaft } \\
\text { Erythrozyten) }\end{array}$ & 2 & nd \\
\hline LP 3 & 355 & $\begin{array}{l}4 \\
\text { (81\% Lymphozyten) }\end{array}$ & 2 & 7,2 \\
\hline
\end{tabular}
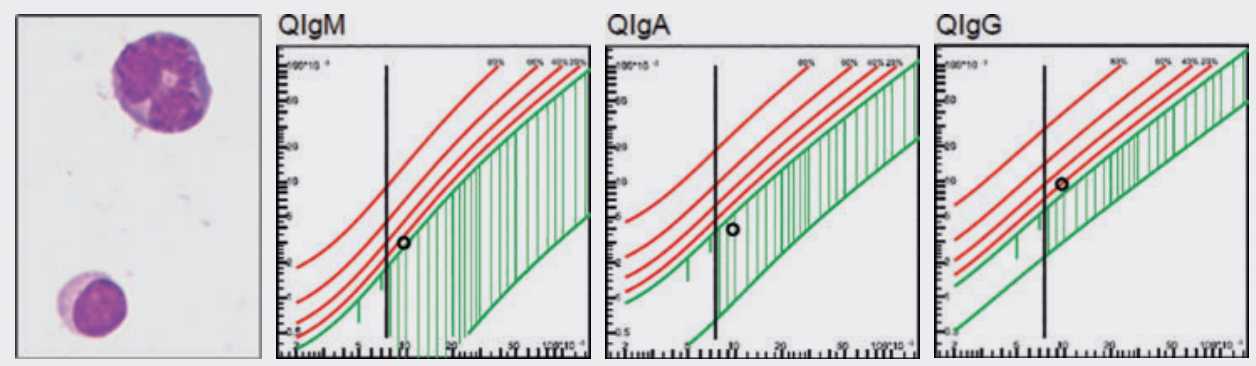

\section{Proteine:}

\begin{tabular}{|l|l|l|l|l|}
\hline Liquor & CSF $\mathbf{~ m g / l}$ & Serum g/l & Q (Liq/Ser) $)^{*} 10^{-3}$ & lok. Synthese \% \\
\hline Albumin & 400.0 & 41.3 & 9.7 & \\
\hline $\lg G$ & 99.3 & 10.5 & 9.457 & 30.68 \\
\hline $\lg \mathrm{A}$ & 9.2 & 2.35 & 3.915 & keine Synthese \\
\hline $\operatorname{IgM}$ & 3.1 & 1.04 & 2.98 & 10.90 \\
\hline
\end{tabular}

- Abb. 1 Initiale Liquorpunktion: Nachweis eines typischen Lymphozyten und eines atypisch-aktivierten Monozyten (links obere Reihe). Nachweis einer Blut-Liquor-Schrankenstörung mit rechnerischem Nachweis einer intrathekalen IgG- und IgM-Synthese (Reiber-Diagramm, obere und untere Reihe). Zusätzlich Nachweis oligoklonaler Banden im Liquor (ohne Abbildung).

Serologisch auffällig war ein erhöhter TPPA-Titer (20 480; $n<80)$, ein erhöhter VDRL-Titer $(8 ; n<2)$ und ein erhöhter FTA-Abs (1600; $n<5)$. In der daraufhin durchgeführten Stufendiagnostik war im Serum der Treponema pallidum IgG blot positiv für Tp47, TmpA, Tp257, Tp453, Tp17 und
Tp15, aber negativ im Treponema pallidum IgM blot. Im Liquor war der Treponema pallidum IgG blot ebenfalls positiv für Tp47, TmpA, Tp257 und Tp17 (grenzwertig positiv für Tp453 und Tp15), allerdings negativ im Treponema pallidum IgM blot ( $\triangleright$ Abb. 2). Der Treponema pallidum IgG 


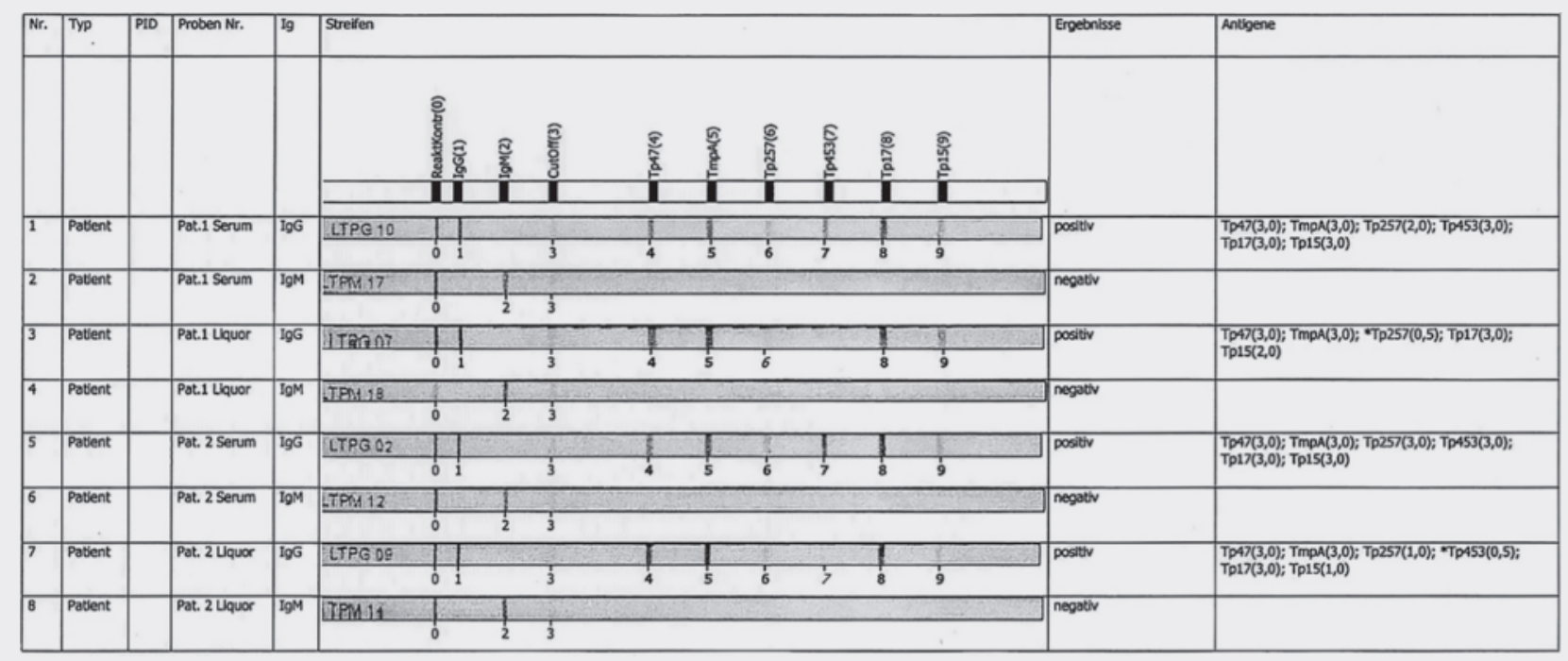

- Abb. 2 Treponema-pallidum-Immunoblot (recomLine IgG/lgM; Mikrogen). Nachweis einer Antikörperbildung gegen wesentliche Antigene von Treponema pallidum sowie Nachweis der Zugehörigkeit der reagierenden Antikörper zur Immunglobulinklasse IgG. Ein mit Treponema-Antigenen beladener Teststreifen wird mit verdünntem Serum oder Plasma inkubiert. Dem Ansatz werden Peroxidase konjugierte antihuman Antikörper (IgG- bzw. IgM-spezifisch) zugegeben. Nach Zugabe der Färbelösung entstehen an den mit Antikörpern besetzten Stellen der Teststreifen unlösliche Farbbanden.

blot fiel somit im Serum und Liquor positiv aus, der -lgMBlot war im Serum und Liquor negativ. Der TPPA-AK-Index war erhöht $(5,3 ; n<2)$. Nach Bebrütung des Liquors konnte kein Wachstum von Bakterien oder Pilzen nachgewiesen werden.

Aus mikrobiologisch-infektiologischer Sicht bestand somit der dringende Verdacht auf eine Neurolues, klinisch wurde die Diagnose einer behandlungsbedürftigen Syphilisinfektion im Quartärstadium mit ZNS-Beteiligung gestellt. Bei fraglicher Penicillinallergie wurde mit Cephalosporinen der dritten Generation (Ceftriaxon $2 \mathrm{~g} / \mathrm{d}$ ) behandelt. Konsiliarische Vorstellungen in der Haut-, Augenklinik sowie Gynäkologie ergaben keine Auffälligkeiten. Ergänzende mikrobiologische Untersuchungen (PCR für Gonokokken, Chlamydia trachomatis aus Zervixabstrich) wurden durchgeführt. Die Untersuchung des Serums auf Chlamydia erbrachte den Nachweis von Antikörpern gegen Chlamydia trachomatis und Chlamydia pneumoniae (speziesübergreifenden Kreuzreaktivitäten). Im Rahmen der Chlamydien-Stufendiagnostik wurde ein Abstrich von der Zervix entnommen, der in der Chlamydia trachomatis PCR positiv war. Die zusätzliche mikrobiologische Untersuchung erbrachte keinen weiteren Hinweis auf Koinfektion mit anderen neurotropen Erregern (HIV ELISA negativ, FSME IgG Antikörperindex 0,6, FSME IgM serologisch negativ. EBV IgM negativ; CMV IgG positiv, -IgM negativ; HSV IgG positiv, -IgM negativ; Borrelia IgG/IgM negativ). Die Hepatitis-Serologie war bei genannter Patientin und ihrem Lebenspartner unauffällig. Im ausführlichen Gespräch mit der Patientin konnte der Infektionszeitpunkt retrospektiv nicht bestimmt werden. Es wurde die Mitbehandlung des
Lebenspartners angeraten. Nach 7 Tagen Antibiose mit Cephalosporin erfolgte eine Kontroll-LP.

Bei weiterhin erhöhter Gesamtzellzahl wurde eine Fortsetzung der parenteralen Cephalosporin-Antibiose für weitere 3 Wochen durchgeführt. Der 52-jährige Partner der Patientin wurde einbestellt und ebenfalls mitbetreut. Laborchemisch zeigte sich bei dem Partner der Patientin ein jeweils erhöhter TPPA- (20 480; $n<80)$, VDRL- (16; $n<2)$, FTA-Titer (1280; $\mathrm{n}<16)$ und TPPA-AI $(19,6)$. Dieser Befund spricht laborseitig für eine Neurolues. In der Liquorpunktion des Patienten war das Gesamtprotein leicht erhöht, ebenso war die Gesamtzellzahl leicht erhöht. Es zeigte sich eine granulozytäre Zellreaktion und Nachweis von massenhaft Erythrozyten (makroskopisch blutigem Liquor bei artifizieller Liquor-Blutbeimengung). Ein entzündlicher ZNS-Prozess konnte nicht sicher ausgeschlossen werden. Die antibiotische Therapie mit Penicillin G (24 Mio. Einheiten/Tag i. v., über 14 Tage) wurde vom Patienten komplikationslos vertragen. In der Kontroll-Liquorpunktion zeigte sich eine unauffällige Gesamtzellzahl und kein rechnerischer Nachweis einer intrathekalen Immunglobulinsynthese bei weiterhin erhöhtem TPPA-Titer (2560; $\mathrm{n}<80)$, VDRL Titer $(2, \mathrm{n}<2)$ und TPPA AI 7,2. Klinisch bot der Partner der Patientin auffällige Befunde in der elektrophysiologischen Untersuchung ( $\triangleright$ Abb. 3, \ Abb. 4). So zeigten die sensibel evozierten Potenziale beider Arme und Beine Leitungsstörungen mit erhöhter kortikaler Latenz und die motorisch evozierten Potenziale deutlich erhöhte kortikale Latenzen und verzögerte periphere Potenziale im Sinne einer axonal-demyelinisierenden Polyneuropathie ( $\triangleright$ Abb. 3, $\triangleright$ Abb. 4). Am ehesten ist dies auf die Trepone- 

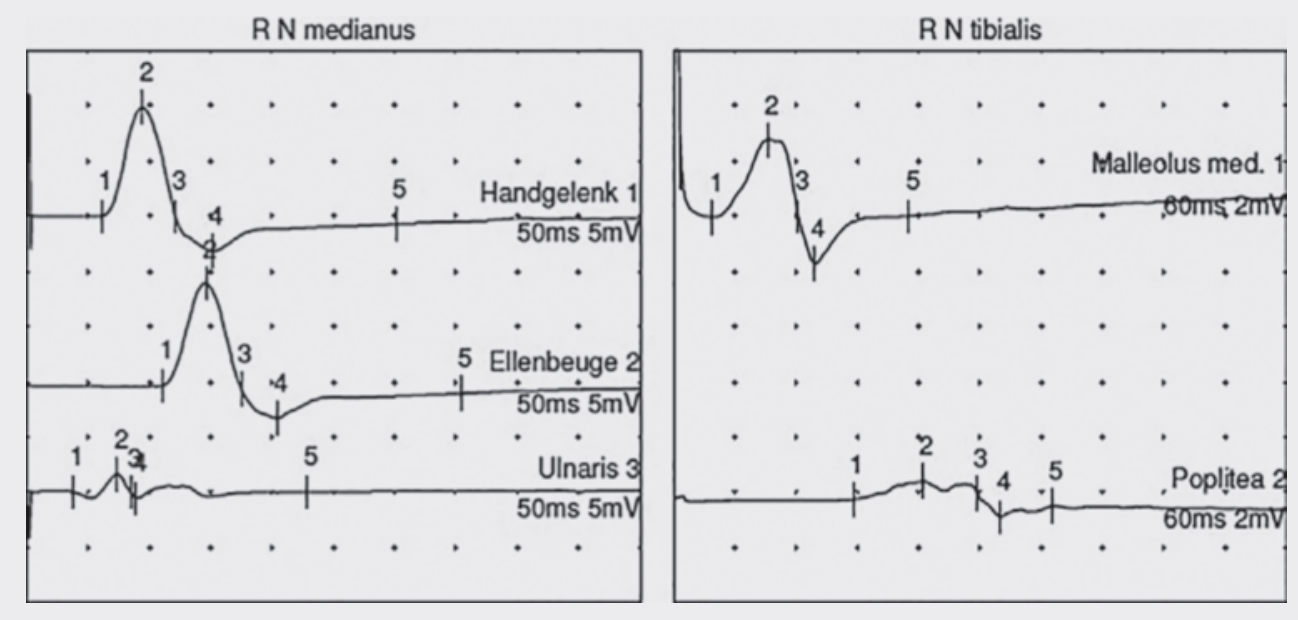

Abb. 3 Motorische Neuropathie mit Amplitudenminderung in der Elektroneurografie bei Tabes dorsalis (Lebenspartner) (Auflösung: 50 ms horizontal/5 mV vertikal)

ma-pallidum-Infektion (Tabes dorsalis) zurückzuführen. Hinweise auf anderweitige Ursachen der Polyneuropathie ergaben sich in der Diagnostik nicht - im Besonderen kein Diabetes mellitus Typ 2 und Alkoholabusus. Eine neuropsychologische Testung wurde von dem Patienten verweigert. Trotz Persistenz der Neurolues lagen bei beiden Patienten subjektiv nur geringfügige bis keine klinischen Symptome vor. Die ausgeprägte kognitive Einschränkung in der neuropsychologischen Testung der Patientin und die deutliche axonal-demyelinisierende Polyneuropathie des Patienten deuten auf eine stattgehabte hohe Aktivität der Infektion trotz oder vor der erstmaligen antibiotischen Therapie hin.

\section{Weiterer Verlauf}

Zwei Jahre später wurde die Patientin erneut nach einem generalisierten epileptischen Anfall zur Abklärung aufgenommen, diesmal bestand eine verzögerte Orientierungsphase und eine passagere Facialisparese links. In der umgehend angefertigten CCT und der ebenfalls durchgeführten zerebralen MRT ergaben sich keine Auffälligkeiten (kein Nachweis einer intrakraniellen Blutung, graue und weiße Substanz ohne pathologischen Befund, unauffällige Weiten der inneren und äußeren Liquorräume, MR-tomografisch kein Anhalt auf einen epileptogenen Fokus oder anderweitige Pathologien). Der extra- und intrakranielle Gefäßstatus konnte sonografisch als unauffällig bewertet werden. Im EEG zeigten sich keine epilepsietypischen Potenziale, aber links frontotemporal eine fokale Verlangsamung als Herdbefund. Bei wiederholtem epileptischem Anfall wurde eine antiepileptische Therapie mit Levetiracetam begonnen. In der Kontrolluntersuchung der Treponema-pallidum-Serologie zeigte sich keine wesentliche Änderung der Befundkonstellation im Vergleich zum Vorbefund (TPPA (10240; $\mathrm{n}<80$ ); VDRL 2; $\mathrm{n}<2$ ), in der Kontrollliquorpunktion zeigte sich weiterhin ein leicht erhöhtes Gesamtprotein (561 mg/l), der Liquor war jedoch zytolo-

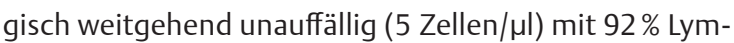
phozyten in der Differenzierung (Glukose 3,1 mmol/l, Laktat $1,1 \mathrm{mmol} / \mathrm{I}$ ) sowie weiterhin eine grenzwertige Blut-Liquor-Schrankenfunktionsstörung mit erneutem Nachweis von zusätzlichen, oligoklonalen Banden im Liquor, vereinbar mit einem chronisch-entzündlichen ZNS-Prozess.

In der Analyse des Serum-Liquor-Paares wurde erneut ein erhöhter Treponema-pallidum-spezifischer AK-Index gemessen (Al 10,3; vierfacher Anstieg im Vergleich zum Vorbefund). Es zeigte sich ein serologischer Rückgang des VDRL-Titers von 8 auf 2 im Vergleich zum Vorbefund ohne Anhalt auf Reinfektion (Treponema pallidum IgM-Blot negativ). Dieser Befund war somit vereinbar mit einer persistierenden Neurolues, obwohl die elektrophysiologischen Untersuchungen, die Bildgebung und die Klinik der Patientin (mit Ausnahme der epileptischen Anfälle und fraglicher Verhaltensauffälligkeiten) nicht richtungsweisend waren. Einzig im EEG bot die Patientin auffällige Allgemeinveränderungen. Bei anamnestisch unklarer Penicillinallergie wurde erstmalig eine 3-malige Gabe (Tag 1, 8 und 15) von Benzathinpenicillin G (2,4 Mio. IU i. m. - verteilt auf 2 Injektionsorte gluteal) durchgeführt, inklusive einer erneuten Partnermitbehandlung. Eine ophthalmologische und gynäkologische Vorstellung sowie die MR-Bildgebung des Schädels und der spinalen Achsen blieben ohne richtungsweisende Befunde. Die dritte Liquorpunktion nach durchgeführter Penicillintherapie erbrachte keine Änderung im Vergleich zu den Vorbefunden. Es wurde daher eine erneute antibiotische Therapie eingeleitet (Penicillin G 24 Mio. Einheiten/Tag i. v. über 14 Tage), welche die Patientin komplikationslos vertrug. In der neuropsychologischen Untersuchung war eine geistige Leistungsminderung mittelschweren Grades nachweisbar, dem Grad einer leichten Demenz entsprechend (Störungen der Flexibilität, des Tempos bei intrinsischen Anforderungen sowie Störungen des Arbeits- und des episodischen Gedächtnis- 

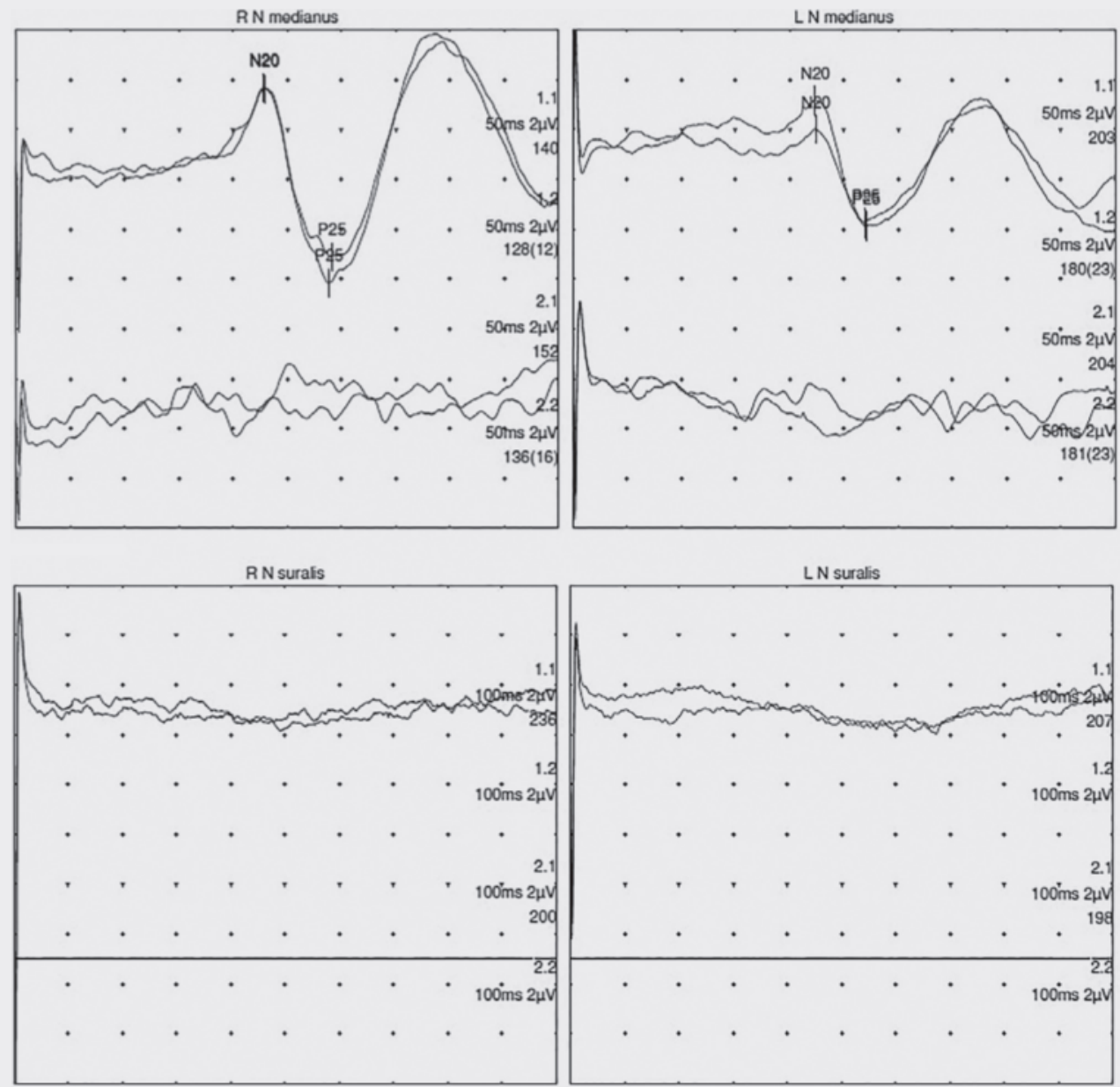

- Abb.4 Sensible Leitungsstörung mit Verlust zentraler und peripherer sensibler Potenziale bei Tabes dorsalis (Lebenspartner) (SEP, Auflösung: 100 ms horizontal $/ 2 \mu \mathrm{V}$ vertikal, antidrome Stimulation Handgelenk und Fußrücken)

ses). Eine Alltagseinschränkung lag jedoch nicht vor. Art und Umfang der Beeinträchtigungen waren den bei Neurolues vorbeschriebenen kognitiven Beeinträchtigungen adäquat [11]. Seit erneuter antibiotischer Therapie und unter Einnahme der angepassten antikonvulsiven Therapie (Lamotrigin $50 \mathrm{mg} / \mathrm{Tag}$ ) war kein erneutes Anfallsereignis mehr aufgetreten. Der gemessene Rückgang der Cardiolipin-AK um mehrere Titerstufen innerhalb eines Jahres wies bei der Patientin auf eine effektive antibiotische Therapie hin ( $\triangleright$ Tab. 1). Insgesamt kann die Befundkonstellation als meningovaskuläre Verlaufsform einer Neurolues gewertet werden, die nur durch wiederholte Antibiotikagaben therapierbar war.

\section{Diskussion und Schlussfolgerung}

Bei generalisierten epileptischen Anfällen, zunehmenden kognitiven Einschränkungen und axonal-demyelinisierender Polyneuropathie sollte eine chronisch-persistierende Treponema-pallidum-Infektion differenzialdiagnostisch erwogen und eine Liquoruntersuchung durchgeführt werden. Der Nachweis einer Pleozytose mit erhöhtem Ge- samtprotein im Liquor, eine Blut-Liquor-Schrankenstörung und eine IgG-dominante Immunreaktion im ZNS sind hinweisend auf eine Neurosyphilis [10] und sollten eine entsprechende Treponema-pallidum-Stufendiagnostik nach sich ziehen. Bei einer gesicherten Neurolues sollte leitliniengerecht antibiotisch therapiert werden (parenterale Gabe von Penicillin G in hoher Dosis 4 Mio. IE alle 4 Stunden über 14 Tage, alternativ parenteral $2 \mathrm{~g} / \mathrm{d}$ Ceftriaxon Initialdosis $4 \mathrm{~g} / \mathrm{d}$ über 10-14 Tage), eine Partnermitbehandlung sollte nicht vergessen werden. Klinische Kontrolluntersuchungen nach der Behandlung werden alle 3 Monate empfohlen [12]. Eine erfolgreiche Behandlung syphilitischer ZNS-Komplikationen sind erkennbar an einem Rückgang von Liquorpleozytose und des VDRL-Titers (Titerabfall um 3-4 log Stufen), an einer Normalisierung der Blut-Liquor-Schranke und der neurologischen Symptomatik innerhalb weniger Monate. Bei Persistenz einer Neurolues können die neurologischen Symptome subjektiv nur geringfügig ausgeprägt sein. Ausgeprägte kognitive Einschränkungen in der neuropsychologischen Testung können retrospektiv auf eine stattgehabte hohe Aktivität der Neurolues hindeuten. 


\section{Interessenkonflikt}

Die Autoren geben an, dass kein Interessenkonflikt besteht.

Danksagung

Die Autoren danken Claudia Helgert, Kerstin Drexler und Katrin Dobschal, Institut für Medizinische Mikrobiologie, Universitätsklinikum Jena für die technische Unterstützung bei der Generierung der infektionsserologischen Befunde, sowie Ines Herrmann und Susanne Ratka, Institut für Klinische Chemie und Laboratoriumsdiagnostik, Universitätsklinikum Jena, für die technische Unterstützung bei der Liquorzytologie.

\section{Korrespondenzadresse}

Dr. med. Matthias Karrasch

Institut für Klinische Chemie und Laboratoriumsdiagnostik Universitätsklinikum Jena

Am Klinikum 1, 07747 Jena

matthias.karrasch@med.uni-jena.de
[4] Reiber H. Peter JB. Cerebrospinal fluid analysis: disease-related data patterns and evaluation programs. J Neurol Sci 2001; 184(2): 101-22

[5] Reiber H. Peter JB. Cerebrospinal fluid in the diagnosis of multiple sclerosis: a consensus report. J Neurol Neurosurg Psychiatry 1994; 57(8): 897-902

[6] Choe PG, Song JS, Song KH et al. Usefulness of routine lumbar puncture in non-HIV patients with latent syphilis of unknown duration. Sex Transm Infect 2010; 86(1): 39-40

[7] Hamouda O, Bremer V, Marcus U et al. [Epidemiological developments of selected sexually transmitted infections in Germany]. Bundesgesundheitsblatt, Gesundheitsforschung, Gesundheitsschutz 2013; 56: 1600-1608

[8] Schummer C, Schliemann S, Fünfstück V et al. Hautmanifestation bei Spätsyphilis. DMW - Deutsche Medizinische Wochenschrift 2014; 139(38): 1883-1886

[9] Schofer H, Brockmeyer NH, Hagedorn HJ et al. [Syphilis. Guideline of the German Sexually Transmitted Diseases Society for diagnosis and therapy of syphilis]. JDDG 2006; 4 : 160-177

[10] Lapere S, Mustak H, Steffen J.Clinical Manifestations and Cerebrospinal Fluid Status in Ocular Syphilis. Ocular Immunology and Inflammation 2019; 27(1): 126-130

[11] Brinar VV, Habek M. Dementia and white-matter demyelination in young patient with neurosyphilis. Lancet 2006; 368: 2258

[12] AWMF Leitlinie Neurosyphilis, Stand 30.09.2012

Bibliografie

DOI https://doi.org/10.1055/a-0916-1184

Nervenheilkunde 2019; 38: 570-575

(c) Georg Thieme Verlag KG Stuttgart · New York

ISSN 0722-1541

[3] Gelpi A, Gilbertson A, Tucker JD. Magic bullet: Paul Ehrlich, Salvarsan and the birth of venereology. Sex Transm Infect 2015; 91(1): 68-69 
Punkte sammeln auf CME.thieme.de

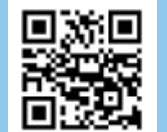

Diese Fortbildungseinheit ist in der Regel 12 Monate online für die Teilnahme verfügbar. Den genauen Einsendeschluss finden Sie unter https://eref.thieme.de/CXD54XP.

Sollten Sie Fragen zur Online-Teilnahme haben, finden Sie unter https://cme.thieme.de/hilfe eine ausführliche Anleitung. Wir wünschen viel Erfolg beim Beantworten der Fragen!

Unter https://eref.thieme.de/CXD54XP oder über den QR-Code kommen Sie direkt zur Startseite des Wissenstests.

VNR 2760512019156645374 trbildu

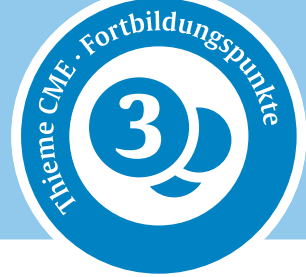

\section{Frage 1}

Welche Aussage ist falsch?

A STD ist eine übliche Abkürzung zur Beschreibung von sexuell übertragbaren Krankheiten, wie beispielsweise der Lues.

B Der Treponema pallidum particle agglutination (TPPA)-Test ist ein Suchtest für Treponema pallidum.

C Der Venereal Disease Research Laboratory (VDRL)-Test ist ein Marker der Entzündungsaktvität einer vorliegenden Syphilis.

D Der Fluoreszenz-Treponemen-Antikörper-Absorption (FTA-Abs)-Test ist ein indirekter Immunfluoreszenztest zum Nachweis Treponema-pallidum-spezifischer Antikörper.

E Bei Verdacht auf Neurolues ist eine Berechnung des Treponema-pallidum-Antikörper-Index (TPP AI) bei positivem TPPA- und VDRL-Test nicht indiziert.

\section{Frage 2}

Welche Aussage zur Diagnostik einer Syphilis ist falsch?

A Der direkte Erregernachweis im Primäraffekt des Stadiums I der Syphilis kann mittels Dunkelfeldmikroskopie geführt werden.

B Spätmanifestationen einer Syphilis im Stadium II/III sind schwer zu diagnostizieren.

C Humanpathogene Treponemen sind schlecht auf bakteriologischen Nährböden kultivierbar.

D Mikrobiologische Untersuchungen hinsichtlich einer STD-Koinfektion (z. B. Gonokokken, Chlamydia trachomatis) sind bei einer gesicherten Neurolues nicht sinnvoll.

E Die Methode der Wahl zur Laboratoriumsdiagnose der Syphilis besteht im Antikörpernachweis (z. B. TPPA-Suchtest, FTA-Abs Bestätigungstest, VDRL).

\section{Frage 3}

Welche Aussage ist richtig?

A Die Therapie der Syphilis wurde erstmalig von Robert Koch in die Klinik eingeführt.

B Bei Neurosyphilis muss zur Erreichung treponemozider Antibiotikaspiegel im Liquor über einen längeren Zeitraum mit Penicillinen und Cephalosporinen therapiert werden.

C Für die Entdeckung des Erregers der Syphilis erhielt Emil Behring den Nobelpreis für Medizin.

D Typische mikrobiologische Befunde einer Neurosyphilis sind erniedrigte TPPA- und VDRL-Titer.

E Die Diagnose einer Neurolues kann im mikrobiologischen Routinelabor unter Anwendung kultureller Verfahren nach Bebrütung des Liquors einfach gestellt werden.

\section{Frage 4}

Welche Aussage zum Treponema-pallidum-Immunoblot ist falsch?

A Ziel ist der Nachweis einer Antikörperbildung gegen wesentliche Antigene von Treponema pallidum.

B Ziel ist der Nachweis der Zugehörigkeit der reagierenden Antikörper zur Immunglobulinklasse IgG.

C Mittels Immunoblot kann die Wirksamkeit, der zur Therapie der Neurosyphilis eingesetzten Cephalosporine bestimmt werden.

D Dem Ansatz werden Peroxidase-konjugierte antihuman Antikörper (IgG-bzw. IgM-spezifisch) zugegeben.

E Nach Zugabe der Färbelösung entstehen an den mit Antikörpern besetzten Stellen der Teststreifen unlösliche Farbbanden. 


\section{Punkte sammeln auf CME.thieme.de}

Fortsetzung $\ldots$

\section{Frage 5}

Welche Aussage ist falsch?

A Zur Diagnosesicherung einer Neurosyphilis sollte eine initiale Liquorpunktion durchgeführt werden.

B Im Liquor kann der Nachweis einer Pleozytose bei gleichzeitig erhöhtem Gesamtprotein hinweisend auf eine Neurosyphilis sein.

C Eine Blut-Liquor-Schrankenstörung ist häufig bei einer Neurosyphilis zu finden.

D Der rechnerische Nachweis einer intrathekalen IgG- und IgM-Synthese kann über das Reiber-Diagramm grafisch dargestellt werden.

E Das gleichzeitige Vorliegen identischer oligoklonaler Banden im Serum und im Liquor beweisen eine Neurosyphilis.

\section{Frage 6}

Die Inzidenz der Syphilis in Deutschland ...

A wird gegenwärtig nicht erfasst, da die Erkrankungshäufigkeit zu gering ist.

B war im Laufe der letzten 10 Jahre stetig steigend, nachdem sie zuvor über einen langen Zeitraum rückläufig war.

C war im Laufe der letzten 15 Jahre stetig sinkend.

D wird nicht erfasst, da die Erkrankung in Deutschland nicht meldepflichtig ist.

E wird nicht erfasst, da die Erkrankung in Deutschland nicht mehr vorkommt.

\section{Frage 7}

Die Goldstandardtherapie der Neurosyphilis basiert auf folgendem Therapieregime:

A Kurzzeitige Hochdosistherapie mit Penicillinen oder Cephalosporinen der dritten Generation.

B Einmalige Depot-Gabe eines Aminopenicillins.

C Langfristige, ggf. mehrfache, intravenöse Therapie mit Penicillinen oder Cephalosporinen der dritten Generation in hoher Dosierung.

D Makrolide in niedriger Dosierung über mindestens 4 Wochen.

E Makrolide in hoher Dosierung über 8 Wochen.

\section{Frage 8}

Welche Aussage trifft nicht zu?

A Die Syphilis wird durch das Bakterium Chlamydia trachomatis ausgelöst.

B Die Diagnose einer Syphilis sollte stets eine Partnermitbehandlung nach sich ziehen.

C Die Syphilis kann über Jahre hinweg klinisch inapperent bleiben.

D Die Syphilis zählt zu den STD.

E Die Neurolues ist eine Spätkomplikation der Syphilis.

\section{Frage 9}

Welche Aussage trifft zu?

A Epileptische Anfälle in Zusammenhang mit einer Neurolues sind stets generalisierte epileptische Anfälle.

B Epileptische Anfälle können als Erstsymptom einer Neurolues auftreten.

C Epileptische Anfälle treten ausschließlich zu Beginn einer Syphiliserkrankung auf.

D Epileptische Anfälle können nur auftreten, solange dermatologische Auffälligkeiten im Genitalbereich nachweisbar sind.

E Epileptische Anfälle können nur bei Patientin mit einer vorbekannten Epilepsie auftreten.

\section{Frage 10}

Welche Aussage ist richtig? Die Tabes dorsalis ist ...

A eine Komplikation der Therapie der Neurolues.

B eine Spätkomplikation der Syphilis.

C ein Anzeichen für eine Remission der Neurolues.

D eine dermatologische Auffälligkeit im Genitalbereich bei Syphilis.

E ein Zeichen einer restitutio ad integrum. 